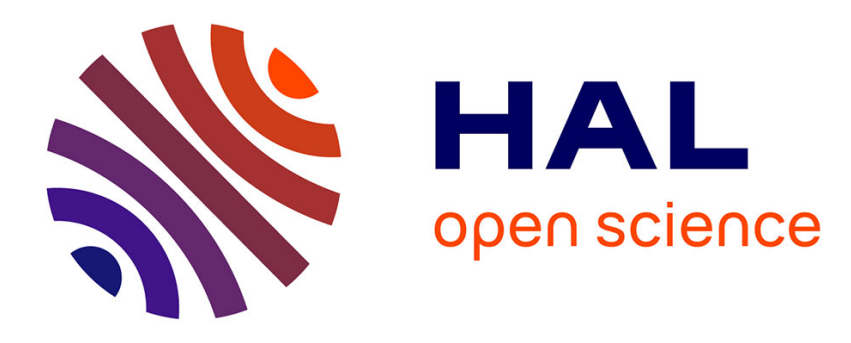

\title{
Multi-mechanism modeling of amorphous polymers
}

\author{
Mohieddine Jeridi, Houda Chouchene, Vincent Keryvin, Kacem Sai
}

\section{To cite this version:}

Mohieddine Jeridi, Houda Chouchene, Vincent Keryvin, Kacem Sai. Multi-mechanism modeling of amorphous polymers. Mechanics Research Communications, 2014, 56, pp.136-142. 10.1016/j.mechrescom.2014.01.003 . hal-00977262

\section{HAL Id: hal-00977262 \\ https://hal.science/hal-00977262}

Submitted on 10 Apr 2014

HAL is a multi-disciplinary open access archive for the deposit and dissemination of scientific research documents, whether they are published or not. The documents may come from teaching and research institutions in France or abroad, or from public or private research centers.
L'archive ouverte pluridisciplinaire HAL, est destinée au dépôt et à la diffusion de documents scientifiques de niveau recherche, publiés ou non, émanant des établissements d'enseignement et de recherche français ou étrangers, des laboratoires publics ou privés. 


\title{
Multi-mechanism modeling of Amorphous polymers
}

\author{
M. Jeridi ${ }^{a}$ H. Chouchene ${ }^{a}$ V. Keryvin ${ }^{b}$ K. Saï ${ }^{a}{ }^{*}$ \\ ${ }^{a}$ LGPMM, Ecole Nationale d'Ingénieurs de Sfax, BP W 3038 Sfax, Tunisia \\ ${ }^{\mathrm{b}}$ LIMATB rue de Saint-Maudé, 56321 Lorient, France
}

\begin{abstract}
The paper is devoted to a multimechanism (MM) model for the mechanical behavior of amorphous glassy polymers. A finite strain formulation through updated lagrangian formalisms is used. In the proposed phenomenological model, three mechanisms are respectively associated to three physical regimes for plastic deformation. The model was successful in describing the stress-strain behavior of glassy polymers for different strain rates and range of temperatures. The description of the three regions observed in the monotonic stress-strain curves is obtained through a coupling matrix between the isotropic hardening variables. A modular strategy based on the determination of the material parameters in three steps is proposed.
\end{abstract}

Key words: Multi-mechanism modeling, Deformation mechanism, Amorphous Polymer, Finite Element

\section{Introduction}

Structural polymers are frequently used in engineering components. The mechanical behavior of glassy polymers has been the subject of considerable research. The literature is abundant in studies devoted to experimental and numerical investigations of mechanical behavior of these polymers. In the following, works related to semi-crystalline polymers are not discussed. Numerous experimental studies have been carried out on polymers to characterize their mechanical behavior in terms of temperature and strain rate sensitivity (Hope et al., 1980; Richeton et al., 2006; Prasad et al., 2009; Nasraoui et al., 2012; Forquin et al., 2012; Cheng and Ghosh, 2013). Various models were developed and tested to simulate these responses. Rheological models extended to three-dimensional case under finite strain assumption have been proposed, for instance, by (Alcoutlabi and MartinezVega, 2003; Anand and Ames, 2006; Dupaix and Boyce, 2007; Ames et al., 2009; Anand et al., 2009; Srivastava et al., 2010; Shim and Mohr, 2011; Fleischhauer et al., 2012; Helbig and Seelig, 2012). Some of them were devoted to phenomenological modeling (Zaïri

\footnotetext{
* Corresponding Author. E-mail adress: kacemsai@yahoo.fr

Preprint submitted to Elsevier Science
}

10 April 2014 
et al., 2005b; Cheng and Ghosh, 2013) or developed within a thermodynamics framework (Drozdov, 1999; Miehe et al., 2009; Bouvard et al., 2013). Studies devoted to constitutive models including damage involves the works of (Zaïri et al., 2005a; Hocine et al., 2011; Zaïri et al., 2011) among others. Some models are focused on predicting the mechanical behavior of amorphous polymers for a wide range of temperatures and strain rates. A non exhautive list of these studies includes the works of (Richeton et al., 2005; Richeton et al., 2007). All these models have a common root in the separation of stress-strain curve into three regimes (Stachurski, 2003): (i) initially, the deformation is nonlinear until a maximum stress, the yield stress, is reached, (ii) the stress decreases due to softening even in absence of necking (iii) molecular orientation provides a mechanism for hardening that predominates at large strains.

The purpose of this article is to offer a new point of view for the description of the glassy polymer mechanical behavior. The stress strain curve is described using the so called Multi-Mechanism (MM) approach. This class of models refers also as well to multimechanism, multi-phase or multi-potential. The MM model considered in this work refers to "multi-mechanism" due to the presence of the three regimes discussed above. The model formulation is detailed in section 2 within a finite strain framework. Indeed, the loading strain ranges between $10 \%$ and $150 \%$ in the following simulations. To assess the model reliability, the proposed MM is compared in sections 3, and 4 with experimental data of PolyCarbonate (PC) and Poly(Methyl MetAcrelate) (PMMA) respectively. The PC and PMMA are, with PolyStyrene (PS), the more commonly used glassy polymers. The selected tests, taken from the literature, were performed at different strain rates and different temperatures. All the experimental results consist mainly of compressive tests and are not concerned with necking phenomenon according to the cited references. A detailed identification procedure of the material parameters is shown in section 3 . In section 5 , a FE (finite element) simulations of plane-strain forging experiments at $25^{\circ} \mathrm{C}$, under isothermal conditions, were performed on PC specimens as in the work of (Srivastava et al., 2010). In that section, the material parameters are first identified using the stressstrain curves. The prediction of the model is then compared with experiments in terms of final forged shape.

\section{Modeling}

The proposed approach is based upon the investigation of multi-mechanism and multicriteria models. The proposed model is an extension to the use of three mechanisms of previous "two mechanism-two criteria" models. This general framework includes the models adopted by authors like (Saï et al., 2004) to predict the stress-strain behavior of metals and applied for the case of polymers by (Regrain et al., 2009) to model Polyamide 6 creep behavior and account for the contribution of both amorphous and crystalline phases. A detailed review of such approach can be found in the work of (Saï, 2011). The use of a finite strain formulation through updated lagrangian formalisms is needed to model large-strain deformation of the studied amorphous polymers. The material behavior is based on Green-Naghdi transformation of the stress-strain problem into an "equivalent material referential". This kind of formulation can be applied to materials with tensorial internal variables without modifying the local evolution rules (Ladeveze, 1980). The model 
is described by:

$$
\underset{\sim}{\mathbf{L}}=\underset{\sim}{\dot{\mathbf{F}}} \mathbf{F}^{-1} \quad \underset{\sim}{\mathbf{D}}=\frac{1}{2}\left(\underset{\sim}{\mathbf{L}}+\underset{\sim}{\mathbf{L}}{ }^{T}\right) \quad \underset{\sim}{\mathbf{\Omega}}=\frac{1}{2}\left(\underset{\sim}{\mathbf{L}}-{\underset{\sim}{\mathbf{L}}}^{T}\right)
$$

Where $\underset{\sim}{\mathbf{F}}$ is the deformation gradient, $\mathbf{\sim}$ the rate of deformation, $\mathbf{D}$ the stretch rate and $\Omega$ the rotation rate. The stretch rate tensor is transported into a local rotated referential:

$$
\underset{\sim}{\dot{\mathrm{e}}}=\underset{\sim}{\mathbf{R}^{T}} \underset{\sim}{\mathrm{D}} \underset{\sim}{\mathbf{R}}
$$

where the rotation tensor $\mathbf{R}$ is determined by the polar decomposition of the deformation gradient $\underset{\sim}{\mathbf{F}}=\mathbf{R} \underset{\sim}{\mathbf{U}}$. $\underset{\sim}{\mathbf{R}}$ and $\mathbf{\sim}$ describes respectively a pure rotation and a pure stretch tensor. The integrated strain tensor is decomposed into both elastic and inelastic parts. Thanks to updated lagrangian formulation, constitutive relations can be expressed as in small strain hypothesis. Therefore dealing with the elastic strain tensor is equivalent to a hypoelastic formulation in agreement with a Green-Naghdi stress rate. The stress measure is here the Cauchy stress $\underset{\sim}{\sigma}$ obtained by using the conjugate stress $\underset{\sim}{\mathbf{S}}$ which results from the material behavior integration:

$$
\underset{\sim}{\sigma}=\operatorname{det}^{-1}(\underset{\sim}{\mathbf{F}}) \underset{\sim}{\mathbf{R}} \underset{\sim}{\mathbf{R}} \mathbf{R}^{T}
$$

Under the small deformation assumption, the total strain can be decomposed into an

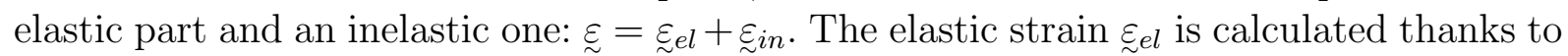
the generalized Hooke's law $: \underset{\sim}{\sigma}=\underset{\sim}{\sim}: \varepsilon_{e l}$ where $\underset{\sim}{\sigma}$ is the Cauchy stress tensor or macroscopic stress resulting from the material behavior integration and $\underset{\sim}{\Lambda}$ is the the fourth-rank tensor of elastic moduli. The inelastic strain $\varepsilon_{i n}$ is the sum of the irreversible deformations of the three observed mechanisms: $\varepsilon_{i n}={\underset{\sim}{v 1}}_{v 1}+{\underset{\sim}{v v 2}}_{v}+\varepsilon_{v 3}$.

In the MM models, each mechanism $I$ is associated to a stress tensor $\sigma_{I}$ calculated from a stress concentration rule. Several approaches were used to obtain $\sigma_{I}$ and strains through different transition rules. It is assumed in the present work that the macroscopic stress $\underset{\sim}{\sigma}$ is equal to the individual stress for the three mechanisms: $\underset{\sim}{\sigma}=\sigma_{1}=\sigma_{2}=\sigma_{3}$. The construction of a constitutive model is in general based on the definition of a yield function, a flow potential and a hardening potential. The proposed model belongs to the MM class model in which each mechanism has its own plastic multiplier. Each stress tensor $\left(\sigma_{1}, \sigma_{\sim}\right.$ and $\left.\sigma_{3}\right)$ is involved in a yield function $\left(f_{1}, f_{2}\right.$ and $\left.f_{3}\right)$ to define the elastic domain related to each mechanism. Since the present study is only dedicated to polymer behavior under monotonic loading, the kinematic hardening variables were not considered.

$$
f_{1}=J(\underset{\sim}{\sigma})-R_{1}-R_{01} \quad f_{2}=J\left(\underset{\sim}{\sigma_{2}}\right)-R_{2}-R_{02} \quad f_{3}=J\left(\underset{\sim}{\sigma_{3}}\right)-R_{3}-R_{03}
$$

Where $J(\underset{\sim}{\sigma})=\sqrt{1.5 \mathbf{s}: \mathbf{s}}$ and $\underset{\sim}{\mathbf{s}}$ is the deviatoric part of the tensor $\underset{\sim}{\sigma} . R_{01}, R_{02}$ and $R_{03}$ denotes the initial size of the elastic domain for the mechanism 1,2 and 3 respectively. The isotropic hardening variables $\left(R_{1}, R_{2}\right.$ and $\left.R_{3}\right)$ that characterize the size change in the elastic domain related to each yield surface are described by three internal variables $\left(r_{1}, r_{2}\right.$ and $\left.r_{3}\right)$ respectively. In this formulation, an isotropic-isotropic coupling has been introduced between the hardening variables to account for the interaction between the 
different mechanisms:

$$
\left(\begin{array}{c}
R_{1} \\
R_{2} \\
R_{3}
\end{array}\right)=\left(\begin{array}{lll}
Q_{11} & Q_{12} & Q_{13} \\
Q_{21} & Q_{22} & Q_{23} \\
Q_{31} & Q_{32} & Q_{33}
\end{array}\right)\left(\begin{array}{c}
r_{1} \\
r_{2} \\
r_{3}
\end{array}\right)
$$

$Q_{11}, Q_{22}$ and $Q_{33}$ are isotropic hardening modulus. $\left(Q_{I J}, I \neq J\right)$ are coupling parameters that play an essential role in the polymer behavior. For example, a positive value of $Q_{12}$ allows to the mechanism 2 (if active) to attenuate the mechanism 1 . Increasing $Q_{12}$ leads to desactivate the mechanism 1. Inversely, a negative value of $Q_{21}$ allows to a premature activation of the mechanism 2 caused by the mechanism 1 . This feature can be helpful, in the case of the studied materials, to activate the third mechanism only after the softening stage. Previous works dealing with MM models have shown that the kinematic coupling allows to describe several mechanical effects (i.e. rate-sensitivity, plasticity-creep interaction, ratcheting, ....).

According to the normality flow rule, the viscoplastic strain rates may be expressed as:

$$
\dot{\sim}_{v 1}=\dot{v}_{1} \mathbf{\mathbf { n }}_{1} \quad{\underset{\sim}{\varepsilon}}_{v 2}=\dot{v}_{2} \mathbf{n}_{2} \quad \stackrel{\sim}{\dot{\varepsilon}}_{v 3}=\dot{v}_{3} \mathbf{n}_{3}
$$

$\dot{v}_{1}, \dot{v}_{2}$ and $\dot{v}_{3}$ are three viscoplastic multipliers and are the driving forces for the evolution laws. They can be written using sinh rules to account for the strong nonlinearity of the creep rate as a function of the stress

$$
\dot{v}_{I}=\dot{v}_{0 I} \sinh \left(\left\langle f_{I} / K_{I}\right\rangle^{n_{I}}\right) \quad I=1,3
$$

The McCauley-brackets \langle\rangle denotes the positive part: $\langle x\rangle=0$ if $x<0$ and $x$ otherwise. $K_{1}$, $n_{1}, K_{2}, n_{2}, K_{3}$ and $n_{3}$ are viscoplastic parameters related to the viscosity of the different mechanisms. The evolution laws of the isotropic hardening variable are given by:

$$
\dot{r}_{1}=\dot{v}_{1}\left(1-b_{1} r_{1}\right) \quad \dot{r}_{2}=\dot{v}_{2}\left(1-b_{2} r_{2}\right) \quad \dot{r}_{3}=\dot{v}_{3}\left(1-b_{3} r_{3}\right)
$$

It is worth noting that the evolution rules of the isotropic internal variables was intentionally simplified compared to the evolution rules that come from thermodynamical consideration as demonstrated in the work of (Saï, 2011) in which "flow" coupling is considered in addition to the "state" coupling (Eq. 5):

$$
\dot{r}_{1}=\dot{v}_{1}\left(1-\frac{b_{1} R_{1}}{Q_{11}}\right) \quad \dot{r}_{2}=\dot{v}_{2}\left(1-\frac{b_{2} R_{2}}{Q_{22}}\right) \quad \dot{r}_{3}=\dot{v}_{3}\left(1-\frac{b_{3} R_{3}}{Q_{33}}\right)
$$

In this work, using the isotropic evolution rules Eq. 8, coupling between the three mechanisms results then only from Eq. 5.

The MM model is implemented into the material library ZMaT of the FE code ZSeT (Besson et al., 1998), using a $\theta$-method solved by an implicit Newton scheme for the local integration. The one-element simulations are shown in sections 3 and 4 using the Green-Naghdi transformation. It is worth noting that the used experimental results are not concerned with necking phenomenon. 


\section{Application to tensile test of PC at room temperature}

To assess the model reliability, the MM model is compared to tensile and relaxation stressstrain curves for a PC at different strain rates at room temperature taken from the work of (Zaïri et al., 2005b). It is important to note that the cited authors did not notice necking in the specimens for the reached strain level $(\varepsilon \simeq 10 \%)$. The Young's modulus $E$ was already determined in the previous work from the initial linear region of the stress-strain curves in which a dependence of $E$ on the strain rate was depicted. It seems therefore necessary to use different coefficients to describe the strain rate effect for the second and the third mechanism. The material constants are determined, in three steps, as follows.

\subsection{Material parameters of the mechanism 1}

The first step of the optimization procedure is devoted to the determination of the material parameters of the mechanism 1 only. Mechanism 1 is supposed to be activated at the beginning of the test so that $R_{01}=0$. Whereas, big values are assigned to the initial size of the elastic domain for mechanisms 2 and $3\left(R_{02} \rightarrow \infty, R_{03} \rightarrow \infty\right)$. The isotropic hardening modulus $Q_{11}$ and the material parameter $b_{1}$ allow to describe the first branch of the tensile curve. The strain rate sensitivity is accounted for thanks to the viscosity parameters $K_{1}$, $n_{1}$ and $\dot{v}_{01}$. These parameters will not be modified beyond this stage. Fig. 1.a shows the superimposition of the experimental results performed by (Zaïri et al., 2005b) and the simulation using the proposed MM model in which only the first mechanism is activated. Figs. 1.b, c and d confirm that only the mechanism 1 is active. The deformation of this mechanism increases fastly and reaches $4 \%$ for the three considered strain rates.

\subsection{Material parameters of the mechanism 2}

The mechanism 2 is devoted to the modeling of the decrease in stress with increasing deformation. The second mechanism is activated by fixing $R_{02}$ to $65 \mathrm{MPa}$ which corresponds almost to the behavior change. The observed softening is modeled by the mean of negative value of the isotropic hardening modulus $Q_{22}$. The rate of this softening seems to be more pronounced then the rate of hardening in the first mechanism, so that $b_{2}=25$. In the contrary, the viscous part of the stress is higher than that observed for the mechanism 1 . This strain rate sensitivity is obtained by the material parameters $K_{2}, n_{2}$ and $\dot{v}_{02}$. Finally, the coupling isotropic hardening modulus $Q_{12}$ and $Q_{21}$ are fixed to zero so that $R_{1}=Q_{11} r_{1}+Q_{13} r_{3}$ and $R_{2}=Q_{22} r_{2}+Q_{23} r_{3}$. The simulated curves after the activation of the mechanism 2 (Fig. 2.a.,b,c and d) show that the deformation of the mechanism 2 is predominant and that the mechanism 1 is attenuated despite the null value of $Q_{12}$.

\subsection{Material parameters of the mechanism 3}

The most difficult task is to activate the third mechanism while decreasing stress for a high value of $R_{03}$. To solve this problem negative values are assigned for the coupling isotropic 
(a)

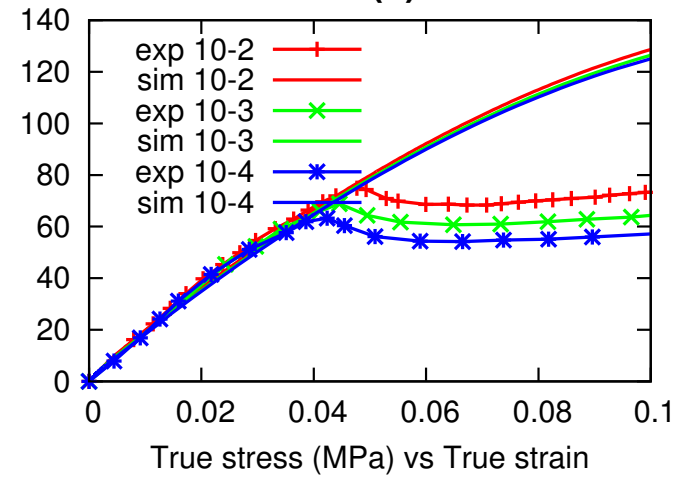

(c)

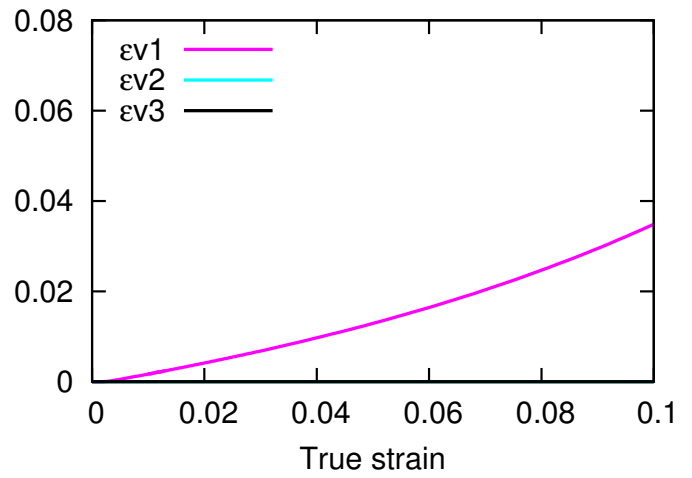

(b)

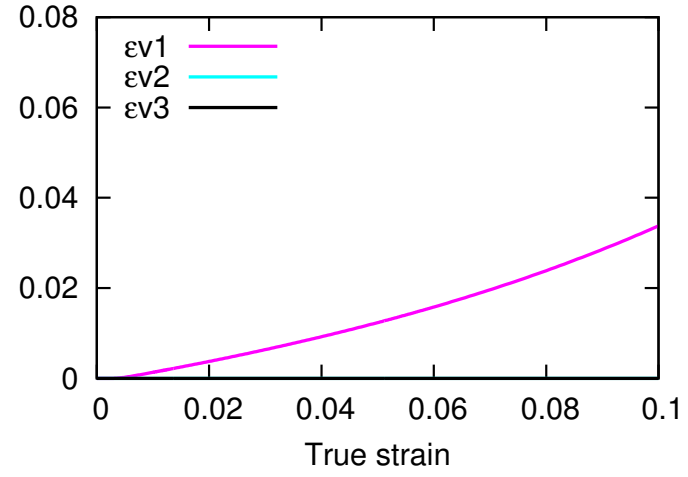

(d)

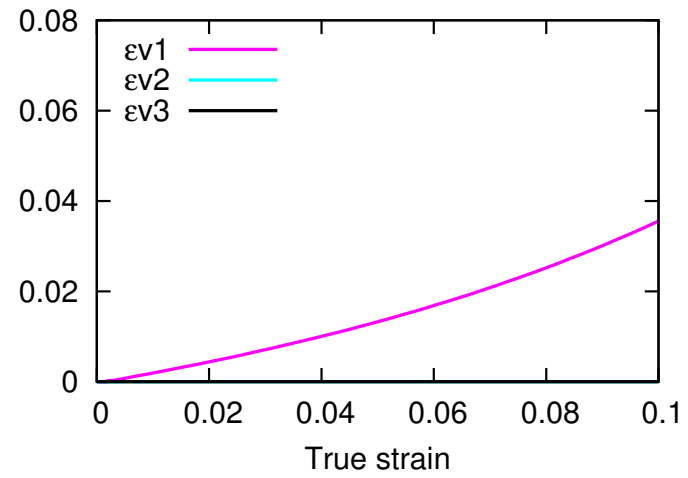

Fig. 1. Application to a Polycarbonate: (a) Experimental, after (Zaïri et al., 2005b), and simulated stress-strain curve if only the first mechanism is activated $\left(R_{02} \rightarrow \infty, R_{03} \rightarrow \infty\right)$. Comparison between viscoplastic deformations of the three mechanism: (b) $10^{-2} s^{-1}$ (c) $10^{-3} s^{-1}$ (d) $10^{-4} s^{-1}$.

hardening modulus $Q_{31}$ and $Q_{32}$. Indeed, the evolution size of the third mechanism is, according to Eq. $5 R_{3}=Q_{31} r_{1}+Q_{32} r_{2}+Q_{33} r_{3}$. $R_{3}$ will be attenuated by the negative terms $Q_{31} r_{1}$ and $Q_{32} r_{2}$. On the other hand, the softening of the mechanism 2 is attenuated by activating the coefficient $Q_{23}$ whereas $Q_{13}$ is kept to be zero. Material parameters are then numerically optimized with a sequential quadratic programming (SQP) method (Stoer, 1985). Optimization consists of minimizing the deviation between experimental data and the simulated ones. The list of the calibrated coefficients for the MM models is given in Tables 1 and the corresponding comparison between simulated responses and experimental data are shown in Fig. 3.a. Figs 3.b, c and d show a decrease of $30 \%$ in the plastic deformation of the second mechanism comparing to Figs 2.b, c and d. It is

Table 1

Identified material parameters of the MM models, Polycarbonate ( $\mathrm{MPa}, \mathrm{mm}, \mathrm{s})$

$\begin{array}{ccccccc}\text { Mechanism 1 } & & K_{1}=50 & n_{1}=5 & \dot{v}_{01}=10 & b_{1}=50 & Q_{11}=8000 \\ \text { Mechanism 2 } & R_{02}=65 & K_{2}=200 & n_{2}=3 & \dot{v}_{02}=25 & b_{2}=25 & Q_{22}=-688.25 \\ \text { Mechanism 3 } & R_{03}=113 & K_{3}=10 & n_{3}=3 & \dot{v}_{03}=25 & b_{3}=5 & Q_{33}=3287.3 \\ \text { Cupling parameters } & & & & Q_{23}=1500 & Q_{31}=-2600 & Q_{32}=-2200 \\ \text { nused parameters } & & R_{01}=0 & Q_{12}=0 & Q_{13}=0 & Q_{21}=0 & \\ \end{array}$

worth noting that, the simulations performed by (Zaïr et al., 2005b) are also in good 
(a)

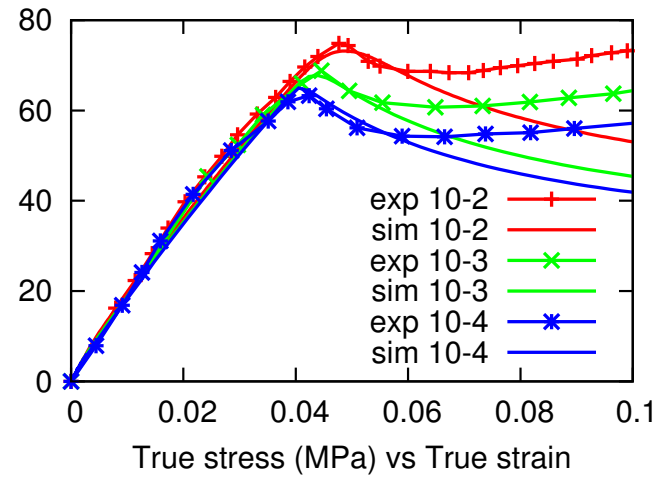

(c)

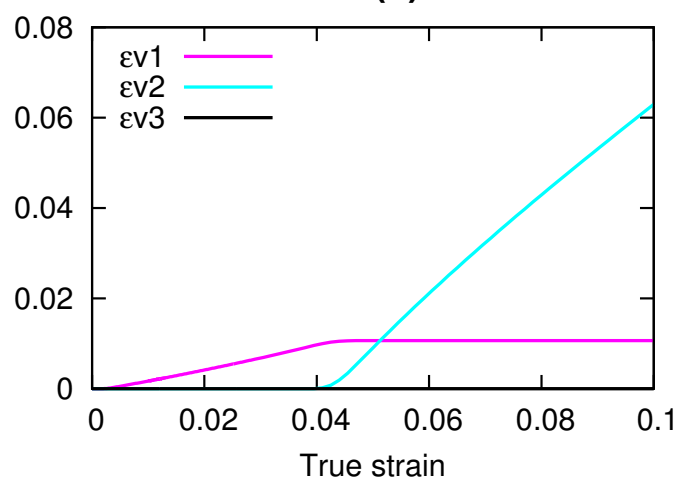

(b)

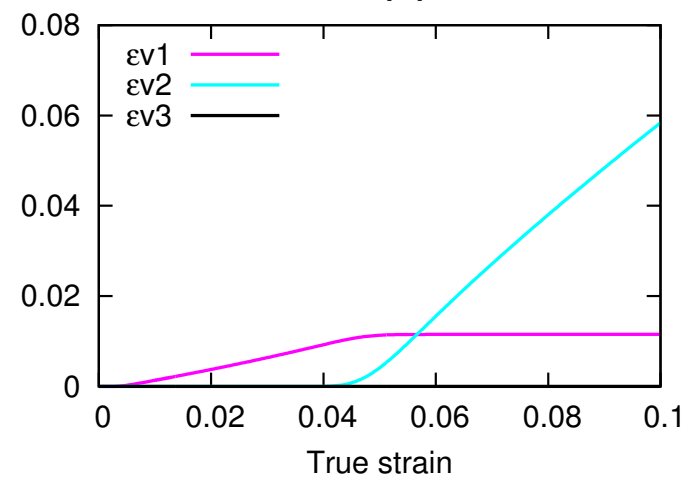

(d)

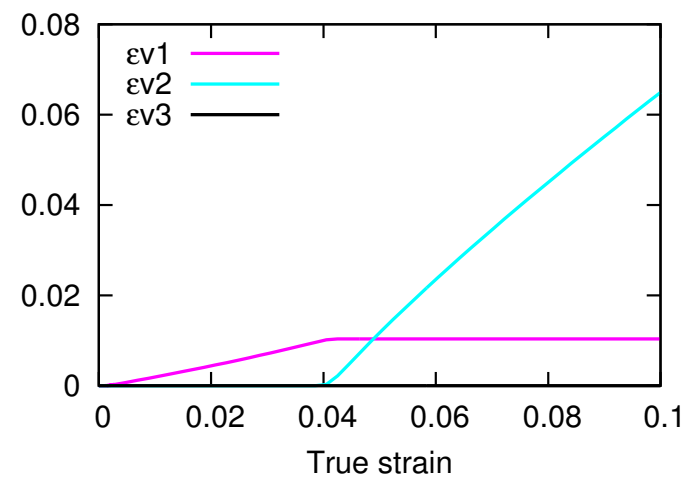

Fig. 2. Application to a Polycarbonate: (a) Experimental, after (Zaïri et al., 2005b), and simulated stress-strain curve if the mechanisms 1 and 2 are activated $\left(R_{03} \rightarrow \infty\right)$. Comparison between viscoplastic deformations of the three mechanism: (b) $10^{-2} s^{-1}$ (c) $10^{-3} s^{-1}$ (d) $10^{-4} s^{-1}$.

agreement with the previous tensile tests. Moreover, two relaxation tests are used to validate a posteriori the prediction of the proposed model. In these tests, the specimens are submitted to a true strain rate of $10^{-3} \mathrm{~s}^{-1}$ followed by stress relaxation with different strain levels. Fig. 4 shows comparison between experiments and prediction of the two models for the relaxation tests.

\section{Application to PMMA with different temperatures}

This section briefly illustrates that the proposed model can reflect the strain rate effect and the temperature effect. Since a time-dependent model is investigated, this is allowed by using material parameters that change as a function of temperature. To assess the model capabilities to quantitatively describe these two effects, experimental data bases obtained on compression tests for PMMA at different temperatures are chosen from the work of (Nasraoui et al., 2012). The list of the identified parameters is given in Tables 2 and the corresponding predicted compression responses for applied strain rates and temperature are shown in Fig. 5. The identification procedure is performed similarily to that described above for each temperature. 
(a)

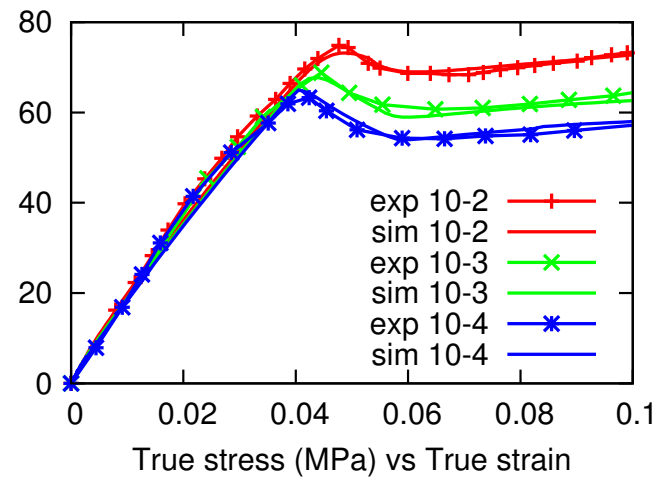

(c)

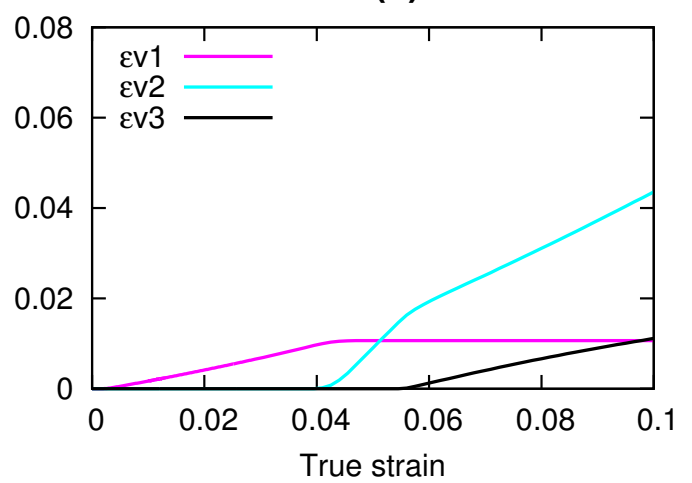

(b)

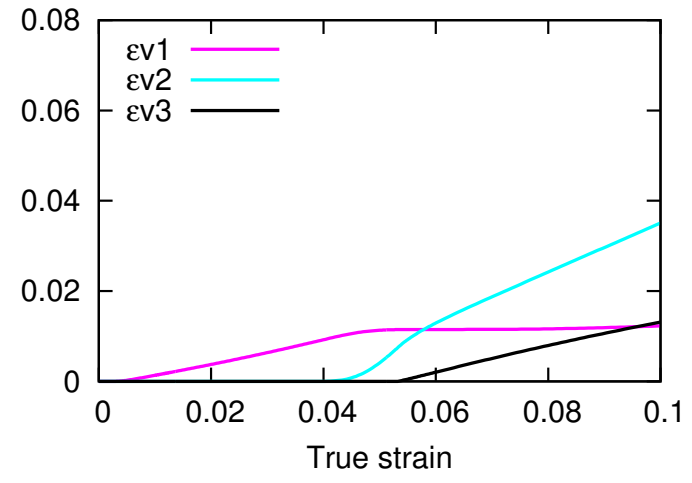

(d)

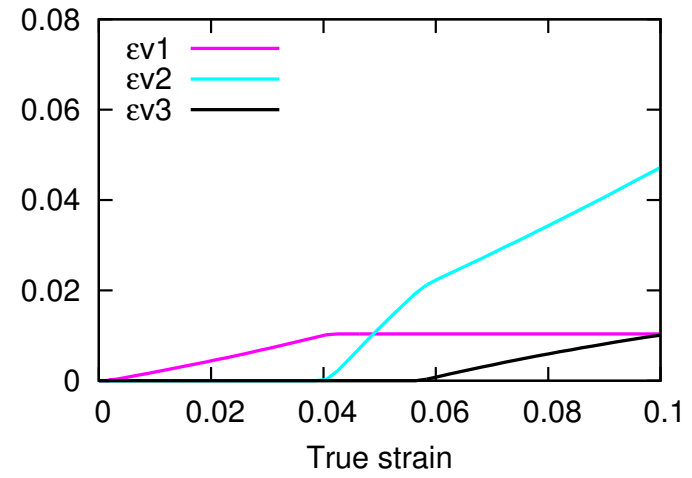

Fig. 3. Application to a Polycarbonate: (a) Experimental, after (Zaïri et al., 2005b), and simulated stress-strain curve if the three mechanisms are activated. Comparison between viscoplastic deformations of the three mechanism: (b) $10^{-2} s^{-1}$ (c) $10^{-3} s^{-1}$ (d) $10^{-4} s^{-1}$.

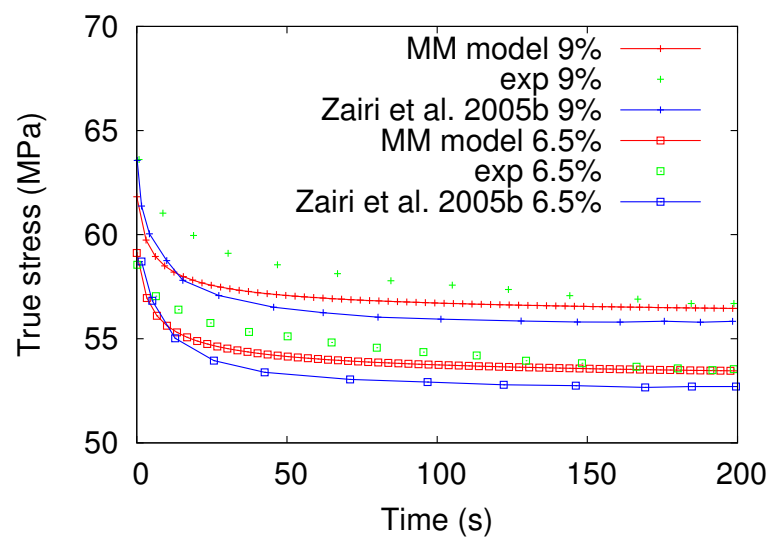

Fig. 4. Validation of the MM proposed model and comparison with two relaxation tests performed by (Zaïri et al., 2005b)

\section{Finite element analysis of plane forging of PC}

In this section, $\mathrm{FE}$ simulations of plane-strain forging experiments at $25^{\circ} \mathrm{C}$, under isothermal conditions, were performed on PC specimens as in the work of (Srivastava et al., 2010). A circular cross-section specimen having a diameter of $12.7 \mathrm{~mm}$ is forged to "cruciform" specimen. The final shape was imparted to the workpiece by a split-dies made of hardened tool steel. The forging experiment was conduced under displacement control to a relative 


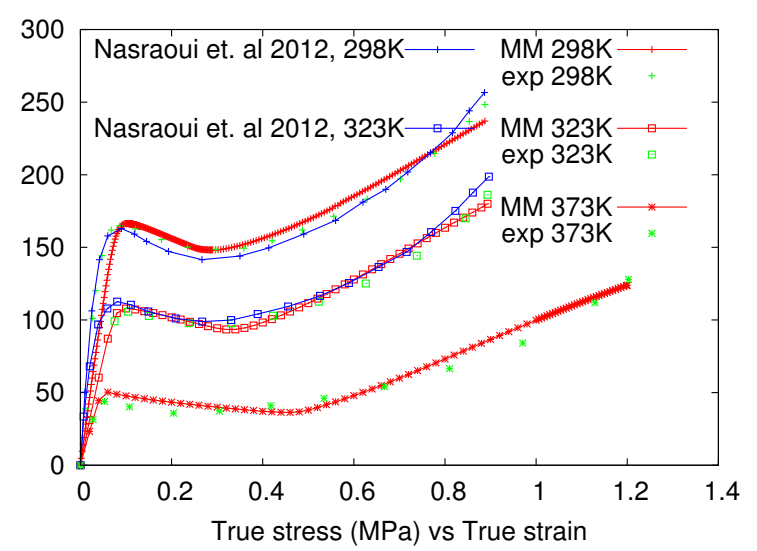

Fig. 5. Comparison with compression tests and simulations performed by (Nasraoui et al., 2012) for a PMMA $\left(\dot{\varepsilon}=10^{-1} \mathrm{~s}^{-1}\right)$.

Table 2

Identified material parameter (MPa, mm, s), PMMA different temperatures.

\begin{tabular}{|c|c|c|c|c|c|c|c|c|c|c|c|}
\hline$T$ & $E$ & $K_{1}$ & $K_{2}$ & $R_{02}$ & $b_{2}$ & $R_{03}$ & $b_{3}$ & $Q_{22}$ & $Q_{23}$ & $Q_{32}$ & $Q_{11}$ \\
\hline $298 \mathrm{~K}$ & 2400 & 230 & 148 & 55 & 10 & 185 & -6 & -32 & 1544 & -1506 & 500 \\
\hline $323 \mathrm{~K}$ & 1600 & 150 & 100 & 37 & 30 & 95 & -9 & -95 & 500 & -10 & 100 \\
\hline $373 \mathrm{~K}$ & 1200 & 70 & 15 & 40 & 47 & 48 & -10 & -70 & 500 & -1000 & 30 \\
\hline$\forall T$ & $n_{1}$ & $n_{2}$ & $K_{3}$ & $n_{3}$ & $b_{1}$ & $Q_{33}$ & $Q_{31}$ & $R_{01}$ & $Q_{12}$ & $Q_{13}$ & $Q_{21}$ \\
\hline & 10 & 26 & 175 & 36 & 5 & 310 & -1230 & 0 & 0 & 849 & 0 \\
\hline
\end{tabular}

die-displacement of $4.6 \mathrm{~mm}$ under displacement rate of $0.02 \mathrm{~mm} / \mathrm{s}$ before the removal of the die at the same absolute rate. Because of the symmetries of the problem, the FE modeling considers only one quarter of the geometry. The plane strain workpiece mesh includes 612 eight nodes elements (C2D8) whereas the die is modeled as a rigid body (Fig. 3.b). The lubrication conditions lead to consider the contact between the die and the workpiece as frictionless. The identification strategy of material parameters is quite different of the procedure described in the previous sections. Comparing to the PC investigated in section 3, a very large strain level is reached so that the hardening mechanisms is extremely pronounced. Accordingly, after some preliminary simulation of the stress-strain curves in which the hardening was underestimated, a new component was added to the isotropic variable $R_{2}$. This additional component is characterized by two coefficient $Q_{2}^{\prime}$ and $b_{2}^{\prime}<0$. Moreover, the kinematic hardening is not taken into account even if loading-unloading stages are considered. The comparison between the simulated and experimental stressstrain behavior is shown in Fig. 6.a. The identified material parameter are displayed in Tab. 3. Fig. 6.d compares the numerically-predicted and experimentally shape taken from the work of (Srivastava et al., 2010) for the studied temperatures after the die removal. A contour plot of the maximal component of the strain tensor at the end of the loading stage is given in Fig. 6.c. Whereas the obtained shape after the die removal is compared successfully in Fig. 6.d with simulation and experiments performed by (Srivastava et al., 2010). 

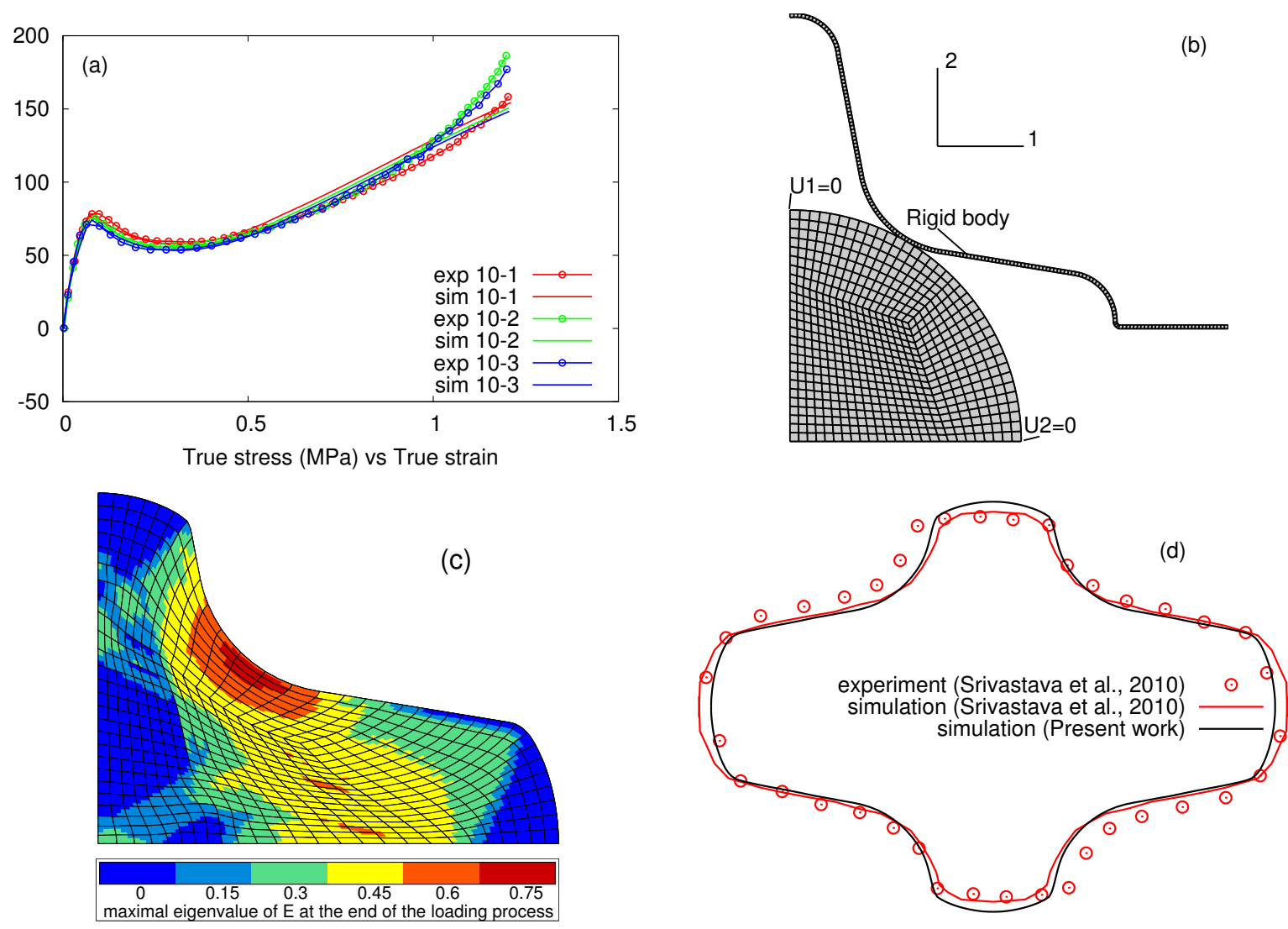

Fig. 6. FE simulation of plane-strain cruciform forging of PC. (a) experimental and simulated stress-strain curves, (b) Quarter symmetry of the FE mesh. (c) Contour plot of the maximal eigenstrain at the end of the loading stage. (d) Comparison between experimentally deformed shape and FE prediction. Experiments are taken from (Srivastava et al., 2010).

Table 3

Identified material parameters of the MM models, PC used in the FE simulation of the cruciform forging $(\mathrm{MPa}, \mathrm{mm}, \mathrm{s})$. Norton law is used for the flow rule. The coupling parameters are fixed to zero.

\begin{tabular}{cccccc} 
Mechanism 1 & $R_{01}=15.5$ & $K_{1}=45$ & $n_{1}=7$ & $b_{1}=9.95$ & $Q_{11}=2079$ \\
Mechanism 2 & $R_{02}=70$ & $K_{2}=13$ & $n_{2}=7$ & $b_{2}=4.3$ & $Q_{22}=-249$ \\
Mechanism 3 & $R_{03}=58$ & $K_{3}=17$ & $n_{3}=7$ & $b_{3}=9.92$ & $Q_{33}=2063$ \\
Additive parameters & $Q_{2}^{\prime}=33$. & $b_{2}^{\prime}=-5.61$ & & & \\
\hline
\end{tabular}

\section{Conclusion}

In this paper, a phenomenological MM model was proposed to describe the mechanical behavior of the amorphous glassy polymers for various strain rates and range of temperatures. The material parameters were calibrated by comparison with experimental data bases taken from the literature. The drawback of this model is the elevated number of material parameters. Nevertheless, it has been shown that these parameters can be split into three groups and can be determined separately according to a modular procedure. The obtained results are very promising and encouraging to explore mechanical behavior in other experimental data bases. In particular cyclic nonlinear behavior of a glassy polymer can be investigated by considering coupling between kinematic hardening variables to 
account for ratcheting phenomenon encountered in the PMMA. On the other hand qualitative experimental observations are needed to check the level of the individual strain of each mechanism predicted by the MM model.

The proposed MM model is implemented in the FE element code Zebulon (Besson et al., 1998). In the present state, it is possible to use it as the constitutive equations in the FE method to analyze inelastic behaviors of components made of glassy polymers submitted to multi-axial loadings under different temperatures.

\section{References}

Alcoutlabi, M. and Martinez-Vega, J. (2003). Modeling of the viscoelastic behavior of amorphous polymers by the differential and integration fractional method: the relaxation spectrum $\mathrm{h}(\tau)$. Polymer, 44:7199 - 7208.

Ames, N. M., Srivastava, V., Chester, S. A., and Anand, L. (2009). A thermo-mechanically coupled theory for large deformations of amorphous polymers. part ii: Applications. Int. J. Plasticity, 25:1495 - 1539.

Anand, L. and Ames, N. (2006). On modeling the micro-indentation response of an amorphous polymer. Int. J. Plasticity, 22:1123 - 1170.

Anand, L., Ames, N. M., Srivastava, V., and Chester, S. A. (2009). A thermo-mechanically coupled theory for large deformations of amorphous polymers. part i: Formulation. Int. J. Plasticity, 25:1474-1494.

Besson, J., Leriche, R., Foerch, R., and Cailletaud, G. (1998). Object-oriented programming applied to the finite element method. Part II. application to material behaviors. Rev. Eur. EF, 7:567-588.

Bouvard, J., Francis, D., Tschopp, M., Marin, E., Bammann, D., and Horstemeyer, M. (2013). An internal state variable material model for predicting the time, thermomechanical, and stress state dependence of amorphous glassy polymers under large deformation. Int. J. Plasticity, 42:168 - 193.

Cheng, J. and Ghosh, S. (2013). Computational modeling of plastic deformation and shear banding in bulk metallic glasses. Comp. mat. sc., 69:494-504.

Drozdov, A. (1999). Modelling the nonlinear viscoelastic behavior of amorphous glassy polymers. Math. Comp. Mod., 30:49-72.

Dupaix, R. B. and Boyce, M. C. (2007). Constitutive modeling of the finite strain behavior of amorphous polymers in and above the glass transition. Mech. Mater., 39:39 - 52.

Fleischhauer, R., Dal, H., Kaliske, M., and Schneider, K. (2012). A constitutive model for finite deformation of amorphous polymers. Int. J. Mech. Sc., 65:48-63.

Forquin, P., Nasraoui, M., Rusinek, A., and Siad, L. (2012). Experimental study of the confined behaviour of PMMA under quasi-static and dynamic loadings. Int. J. Impact Eng., 40 - 41:46 - 57.

Helbig, M. and Seelig, T. (2012). Micro-mechanical modeling of fibrillation in amorphous polymers. Comp. mat. sc., 52:118-122.

Hocine, N. A., Hamdi, A., Abdelaziz, M. N., Heuillet, P., and Zaïri, F. (2011). Experimental and finite element investigation of void nucleation in rubber-like materials. Int. J. Solids Struct., 48:1248 - 1254.

Hope, P., Ward, I., and Gibson, A. (1980). The hydrostatic extrusion of polymethylmethacrylate. J. Mat. Sc., 15:2207-2220. 
Ladeveze, P. (1980). Sur la théorie de plasticité en grandes déformations. ENS-CachanLMT, Internal Report No 9.

Miehe, C., Göktepe, S., and Diez, J. M. (2009). Finite viscoplasticity of amorphous glassy polymers in the logarithmic strain space. Int. J. Solids Struct., 46:181-202.

Nasraoui, M., Forquin, P., Siad, L., and Rusinek, A. (2012). Influence of strain rate, temperature and adiabatic heating on the mechanical behaviour of poly-methylmethacrylate: Experimental and modelling analyses. Materials and Design, 37:500 509.

Prasad, K. E., Keryvin, V., and Ramamurtya, U. (2009). Pressure sensitive flow and constraint factor in amorphous materials below glass transition. J. Mater. Res., $24: 865-872$.

Regrain, C., Laiarinandrasana, L., Toillon, S., and Saï, K. (2009). Multi-mechanism models for semi-crystalline polymer: Constitutive relations and finite element implementation. Int. J. Plasticity, 25:1253 - 1279.

Richeton, J., Ahzi, S., Daridon, L., and Rémond, Y. (2005). A formulation of the cooperative model for the yield stress of amorphous polymers for a wide range of strain rates and temperatures. Polymer, 46:6035 - 6043.

Richeton, J., Ahzi, S., Vecchio, K., Jiang, F., and Adharapurapu, R. (2006). Influence of temperature and strain rate on the mechanical behavior of three amorphous polymers: Characterization and modeling of the compressive yield stress. Int. J. Solids Struct., $43: 2318-2335$.

Richeton, J., Ahzi, S., Vecchio, K., Jiang, F., and Makradi, A. (2007). Modeling and validation of the large deformation inelastic response of amorphous polymers over a wide range of temperatures and strain rates. Int. J. Solids Struct., 44:7938 - 7954.

Saï, K. (2011). Multi-mechanism models: Present state and future trends. Int. J. Plasticity, 27:250 - 281.

Saï, K., Aubourg, V., Cailletaud, G., and Strudel, J. (2004). Physical basis for model with various inelastic mechanisms for nickel base superalloy. Mat. Sc. Tech., 20:747-755.

Shim, J. and Mohr, D. (2011). Rate dependent finite strain constitutive model of polyurea. Int. J. Plasticity, 27:868-886.

Srivastava, V., Chester, S. A., Ames, N. M., and Anand, L. (2010). A thermomechanically-coupled large-deformation theory for amorphous polymers in a temperature range which spans their glass transition. Int. J. Plasticity, 26:1138 - 1182.

Stachurski, Z. (2003). Strength and deformation of rigid polymers: the stress-strain curve in amorphous PMMA. Polymer, 44:6067 - 6076.

Stoer, J. (1985). Principles of sequential quadratic programming methods for solving nonlinear programs (ed. K. Schittkowski). NATO ASI Series, vol. 15, Springer Verlag, Berlin.

Zaïri, F., Naït-Abdelaziz, M., Gloaguen, J., and Lefebvre, J. (2011). A physically-based constitutive model for anisotropic damage in rubber-toughened glassy polymers during finite deformation. Int. J. Plasticity, 27:25 - 51.

Zaïri, F., Naït-Abdelaziz, M., Woznica, K., and Gloaguen, J.-M. (2005a). Constitutive equations for the viscoplastic-damage behaviour of a rubber-modified polymer. Eur. J. Mech. - A/Solids, 24:169-182.

Zaïri, F., Woznica, K., and Naït-Abdelaziz, M. (2005b). Phenomenological nonlinear modelling of glassy polymers. Comptes Rendus Mécanique, 333:359 - 364. 